\title{
Is NAFLD a Risk Factor for Colorectal Polyps? An Analysis of Single Romanian Centre
}

\author{
Kozma Bela*, Pop Crina, Petrut Madalina, Drasovean Silvia, Dobru Daniela \\ Department of Gastroenterology, Mures County Hospital, University of Medicine and Pharmacy of Tîrgu Mureș, Romania
}

\begin{abstract}
Background and Aim: Non-alcoholic fatty liver disease (NAFLD) is considered to be the most common of all liver disorders, and its prevalence is almost certainly increasing. Because of the presence of NAFLD in metabolic syndrome and of latter's role in the appearance of colorectal polyps, many studies have tried to find a link between the liver disease and colorectal polyps. The aim of the study was to determine whether NAFLD is a risk factor for colorectal polyps.

Material and Methods: We examined 560 patients diagnosed with NAFLD who underwent colonoscopy at Mures County Hospital, between January 2011 and June 2014. Fatty liver disease was assessed by abdominal ultrasound, with NAFLD defined as fatty liver disease in the absence of alcohol use of $>40 \mathrm{~g} /$ week or other secondary causes. We divided the 560 patients into two groups, according to the presence or absence of polyps. Data were collected from patient's history, physical exam, laboratory tests and abdominal ultrasound.

Results: The prevalence of colorectal polyps was 16.6\% (93) in the NAFLD patients. 51 (54.8\%) of them were female, and the Chi2 test identified a statistically significant association between the female gender and the appearance of polyps $(\mathrm{p}=0.028 ; \mathrm{OR}=1.652 ; \mathrm{Cl}=1.052-2.596)$. No statistical association was found between the smoking status and the development of colorectal polyps ( $p=0.245)$ and only 18 of 93 patients were smokers. Of 93 patients with polyps, 68 (73.1\%) have associated diabetes mellitus, but without statistical correlation ( $p=0.214)$. According to family history of colorectal polyps none of the patients have data about a possible polyposis pathology.

Conclusions: Our study demonstrates a relationship between some characteristics of NAFLD and the development of colorectal polyps. Further studies are required to confirm whether NAFLD is a risk factor for the appearance of polyps.
\end{abstract}

Keywords: NAFLD, colorectal polyps, abdominal ultrasound, colonoscopy

Received: 09 december 2014 / Accepted: 03 June 2015

\section{Introduction}

Colorectal cancer (CRC) is the third most common cancer worldwide and one of the leading causes of death in Europe [1]. Colorectal adenomatous polyps are considered as precursors of invasive colorectal adenocarcinoma [2]. In addition to its alarming growth rate among the general population, the identification of risk factors for increased propensity of colorectal neoplasms has placed this disease high on the priority list of health care professionals. Metabolic syndrome (MS) increases the risk of colorectal cancer through various biological mechanisms, particularly those related to insulin resistance[3]. Metabolic syndrome is by definition present whenever at least three of the following factors are present: increased waist circumference( $\geq 100 \mathrm{~cm}$ in men, $\geq 90 \mathrm{~cm}$ in women), hipertriglyceridemia $(\geq 150 \mathrm{mg} / \mathrm{dl})$, low high-density lipoprotein (HDL) cholesterol ( $\leq 50 \mathrm{mg} / \mathrm{dl}$ in women and $\leq 40 \mathrm{mg} / \mathrm{dl}$ in men), hypertension $(\geq 130 / 85 \mathrm{mmHg})$, and fasting glucose ( $\geq 105 \mathrm{mg} / \mathrm{dl}$ ). Nonalcoholic fatty liver disease (NAFLD) is currently not a component of the diagnostic criteria for metabolic syndrome, but is also recognized that NAFLD is the hepatic manifestation of metabolic syndrome [4] and represents the most frequent from all liver disorders, with an increasing prevalence, which is reported nowadays to be as high as $20-46 \%$ in Western countries [5]. Kim and

* Correspondence to: Bela Lorand Kozma

E-mail: drkozmabela@gmail.com colleagues found that in patients with metabolic syndrome the prevalence of colonic adenomatous polyps was $17 \%$, compared with a prevalence of $11 \%$ in population without MS. sysyndrome.

The aim of the study was to determine whether NAFLD is a risk factor for colorectal polyps.

\section{Material and Methods}

We performed a prospective observational study on 560 patients diagnosed with NAFLD who underwent colonoscopy at Mures County Hospital, between January 2011 and June 2014. Study participants were previously enrolled in a NAFLD diagnosis study.

The study protocol included the following information: demographic data (age, gender), the presence / absence of colorectal polyps, smoking status, body mass index (BMI) and the presence of diabetes mellitus (DM), hypertension and metabolic syndrome. Data from patient's history, physical exams, laboratory tests and abdominal ultrasound were collected and they were divided into two groups: a) NAFLD patients with colorectal polyps and b) NAFLD patients without colorectal polyps.

\section{Laboratory assessment}

Full blood counts were obtained in all subjects by standard laboratory methods. Blood was centrifuged, and plasma was analyzed for aspartate aminotranspeptidase, alanine aminotranspeptidase, gamma-glutamyl transpeptidase, levels 
of triglycerides, total cholesterol, fasting glucose and uric acid concentration.

\section{Ultrasound examination}

All patients underwent abdominal echography, performed by experienced operators, with at least 5 years of experience. Fatty liver was defined as an increase echogenicity as compared with renal parenchyma, attenuation of the ultrasound beam with no distincion of the diaphragm, or low visibility of the portal walls. NAFLD was by definition considered as the presence of fatty liver disease in the absence of alcohol use or in the absence of any other causes (infections, immunological disorders, heredity).

\section{Colonoscopy}

The laxative Picoprep (containing sodium picosulfate 10 $\mathrm{mg}$, magnesium oxide $3,5 \mathrm{~g}$, citric acid $2 \mathrm{~g}$ which together form magnesium citrate in solution; Ferring Pharmaceuticals, Inc.) was used for bowel preparation before colonoscopy. We examined the colonoscopic features, including the location, size, number, attachment and histology of colorectal polyps. The location for polyps was divided into the proximal colon including caecum, ascending colon and transverse colon; distal colon including the splenic flexure, descending colon, sigmoid colon and rectum. The size of the adenomatous polyp was classified into those less than $5 \mathrm{~mm}, 5-9 \mathrm{~mm}$ and $10 \mathrm{~mm}$ or more. The number of adenomatous polyps was classified into one, two and three or more polyps.

The ethical approval was granted by the ethics and scientific review committee of the institution and informed patient consent was taken adequately.

\section{Statistical analysis}

Statistical analysis was performed using the Statistical Package for Social Sciences (SPSS, version 20, Chicago, IL, USA) and Microsoft Access. Data were labeled as nominal and were characterized by means of frequencies. The frequencies of nominal variables were compared with a Chisquare test. Differences in the mean or median between groups were analyzed using the test, Mann-Whitney test, when appropriate. The Spearman rank test was used to assess the correlation between NAFLD and colorectal polyps after adjusting for independent variables previously known to be associated with increased polyp risk, such as age, BMI and family history. The level of statistical significance was set at $\mathrm{p}<0.05$.

\section{Results}

A total of 560 patients diagnosed with NAFLD based on abdominal ultrasound were included in the following analysis. 467 subjects (83.4\%) did not have colorectal polyps on colonoscopy examination and served as the control group while $93(16.6 \%)$ had positive colonoscopy for colorectal polyps. All patients were stratified in relation to their gender, race, BMI and family history. This was followed by adjustment for variables that have been proved to be associated with an increased risk of adenoma.

The mean age was $59.07 \pm 10.96$ years in the control group and $63.98 \pm 8.92$ in the polyp group, with a significant difference between these two groups $(\mathrm{p}=0.00005)$. Fifty-one $(54.8 \%)$ of them were female, and the $\mathrm{Chi}^{2}$ test identified a statistically significant association between the female gender and the appearance of polyps $(\mathrm{p}=0.028$; $\mathrm{OR}=1.652 ; \mathrm{CI}=1.052-2.596)$. Of 560 patients, 155 $(27.7 \%)$ were smokers and 18 subjects associated colorectal polyps. To assess whether tobacco smoking is associated with the neoplasia, $\mathrm{Chi}^{2}$ test was performed, without any statistical relevance $(\mathrm{p}=0.245)$ (Table I).

Patients with NAFLD were significantly older than the control group. Moreover, after regulation for age, patients with NAFLD and polyps had higher mean BMI, systolic and diastolic blood pressure, fasting glucose, total cholesterol and triglycerides concentration than the control group. Clinical and biochemical features of participants with and without colorectal polyps are shown in Table II.

For assessing the correlation between the body mass index (BMI) and the presence of polyps in NAFLD subjects, we divided the patients intro 3 groups: a) BMI: 18.5-24.9: 21 cases b) BMI: 25-29.9: 27 cases and c) BMI: >30: 33 cases. No statistical association was found between BMI and colorectal polyps ( $\mathrm{p}=0.685)$. Of 560 NAFLD patients, 303 were hypertensive but only 36 of them had associated polyps, without a statistical correlation. Of 93 patients with polyps, 68 (73.1\%) have associated diabetes mellitus, but without statistical correlation $(\mathrm{p}=0.214)$. According to family history of colorectal polyps none of the patients have data about a possible polyposis pathology.

Table I. Baseline demographic characteristics

\begin{tabular}{lcc}
\hline Variables & Category & No. $(\%)$ \\
\hline Age group & $35-44$ years & $9.64 \%$ \\
& $45-54$ years & $18.57 \%$ \\
& $\geq 55$ years & $69.82 \%$ \\
\hline Gender & Male & $45.2 \%$ \\
& Female & $54.8 \%$ \\
\hline Smoking status & Nonsmoker & $80.65 \%$ \\
& Current- smoker & $19.35 \%$ \\
\hline Past history & Hypertension & $58.39 \%$ \\
& Diabetes mellitus & $23.57 \%$ \\
\hline
\end{tabular}

Table II. Cardiometabolic parameters conforming to NAFLD

\begin{tabular}{lccc}
\hline & No Polyps & Polyps & P-value \\
\hline Age (years) & $58.11 \pm 11.19$ & $63.98 \pm 8.92$ & 0.00005 \\
Systolic BP $(\mathrm{mmHg})$ & $132.4 \pm 18.63$ & $133.6 \pm 18.89$ & $\mathrm{NS}$ \\
Diastolic BP $(\mathrm{mmHg})$ & $82.5 \pm 10.67$ & $83.3 \pm 9.54$ & $\mathrm{NS}$ \\
Fasting glucose $(\mathrm{mg} / \mathrm{dl})$ & $111.04 \pm 32.41$ & $108.21 \pm 29.55$ & $\mathrm{NS}$ \\
Total cholesterol $(\mathrm{mg} / \mathrm{dl})$ & $208.01 \pm 51.08$ & $214.46 \pm 40.11$ & $\mathrm{NS}$ \\
Triglycerides $(\mathrm{mg} / \mathrm{dl})$ & $129.05 \pm 71.3$ & $138.2 \pm 69.4$ & 0.002 \\
AST $(\mathrm{IU} / \mathrm{l})$ & $28.1 \pm 35.17$ & $26.26 \pm 13.31$ & $\mathrm{NS}$ \\
ALT $(\mathrm{IU} / \mathrm{l})$ & $38.23 \pm 66.83$ & $28.77 \pm 14.91$ & $\mathrm{NS}$ \\
GGT $(\mathrm{IU} / \mathrm{l})$ & $48.37 \pm 60.88$ & $40.32 \pm 36.12$ & $\mathrm{NS}$ \\
Uric Acid (mg/dl) & $4.3 \pm 1$ & $4.8 \pm 0.8$ & $\mathrm{NS}$ \\
\hline BP: blood pressure; NS: not significant; AST: aspartate aminotranspeptidase; ALT: alanine \\
aminotranspeptidase; GGT: gamma-glutamyl transpeptidase; DM: diabetes mellitus.
\end{tabular}


The analysis conducted according to location, size and number of polyps is shown in Table III.

Table III. Colorectal polyps in NAFLD patients

\begin{tabular}{cc}
\hline & NAFLD (N=93) \\
\hline Location & \\
Proximal colon & $35(37.63 \%)$ \\
Distal colon & $58(62.37 \%)$ \\
\hline Size $(\mathrm{mm})$ & \\
$<5$ & $38(40.86 \%)$ \\
$5-10$ & $31(33.34 \%)$ \\
$\geq 10$ & $24(25.8 \%)$ \\
\hline Number & \\
1 & $50(53.76 \%)$ \\
2 & $28(30.10 \%)$ \\
$\geq 3$ & $15(16.14 \%)$ \\
\hline
\end{tabular}

\section{Discussion}

Several epidemiologic studies have shown that metabolic syndrome is associated with an increased risk for colorectal cancer. Currently, non-alcoholic fatty liver disease (NAFLD) is considered to be an integral part of the metabolic syndrome with insulin resistance as a central pathogenic factor. It is considered nowadays that NAFLD may represent a risk factor for development of adenomatous polyps, however little is known about the possible association between colorectal neoplasms and NAFLD [6-8].

In line with previous reports, we found a lower overall rate of colorectal adenomas in male compared with female subjects, hormonal effects may be involved in polyps development.

Associations between colorectal cancer and individual metabolic syndrome components have not been studied extensively, but some studies from literature have demonstrated an association between type II diabetes mellitus (DM), which commonly is associated with the metabolic syndrome and colorectal cancer. These studies have reported that colorectal cancer is associated with insulin resistance, IGF systems, leptin, adiponectin levels, inflammatory and transcription factors $[9,10]$. In the ARIC Study, data suggest an association between insulin resistance and colorectal cancer. Similar to this study, Colangalo et al. and Trevisan et al. reported modest increases in the risk of colorectal cancer mortality, from 1.14-fold to 1.36-fold, over quartiles of systolic blood pressure [11].

Epidemiological studies have shown that high fat diet, alcohol intake, physical inactivity and obesity are associated with the increased risk of colorectal cancer. These factors are positively related with serum lipids and plasma glucose. Many studies demonstrated the role of high level cholester$\mathrm{ol}$ in carcinogenesis, but less information is available on the relationship between the serum triglycerides and colorectal neoplasm. Unfortunately, even if studies have assessed that increased serum lipids are risk factors for colorectal cancer, few data exist regarding the relationship between these risk factors and colorectal adenomatous polyps. Our study clearly indicates that high level triglycerides are associated with the development of adenomatous polyps.

Our study has several limitations. First, the diagnosis of NAFLD was based on ultrasound examination but was not confirmed by a liver biopsy. NAFLD is defined by the presence of macrovesicular fat in more than $5 \%$ of hepatocytes (on liver biopsy), but on ultrasound examination fatty liver (increased echogenicity) is observed when the hepatic cells contain more than $15 \%$ fat. However, the sensitivity of ultrasonography in the diagnosis of fatty liver disease and the correlation of fatty liver disease with histology have been shown to be high [12]. Secondly, the average followup duration in our study, 3 years, may have been too short to observe a sufficient incidence of colorectal polyps.

In conclusion, we found an increased prevalence of colorectal polyps in patients group with NAFLD, compared with the other group without NAFLD. Also, we found an association between older age and the occurrence of colorectal polyps and between the female gender and polyp development. Consequently, these are independent risk factors for the development of polyps and colorectal cancer later. Hipertriglyceridemia was shown to be an independent risk factor for colorectal polyps in NAFLD patients and no association was found between high level cholesterol and polyps, although the mean level of total cholesterol was higher in polyp group.

\section{References}

1. Ferlay J, Soerjomataram I, Dikshit R, et al. Cancer incidence and mortality worldwide: sources, methods and major patterns in GLOBOCAN 2012. Int J Cancer. 2015;136:E359-E386.

2. Tanaka T. Colorectal carcinogenesis: Review of human and experimental animal studies. J Carcinog. 2009;8:5.

3. Touzin NT, Bush KN, Williams CD, Harrison SA. Prevalence of colonic adenomas in patients with nonalcoholic fatty liver disease. Therap Adv Gastroenterol. 2011;4:169-176.

4. Tarantino G., Finelli C. What about non-alcoholic fatty liver disease as a new criterion to define metabolic syndrome?. World J Gastroenterol. 2013;19:3375-3384.

5. Williams CD, Stengel J, Asike MI, etal. Prevalence of nonalcoholic fatty liver disease and nonalcoholic steatohepatitis among a largely middleaged population utilizing ultrasound and liver biopsy: a prospective study. Gastroenterology. 2011;140:124-131.

6. Singh SP, Singh A, Pati GK, et al - A Study of Prevalence of Diabetes and Prediabetes in Patients of Non-Alcoholic Fatty Liver Disease and the Impact of Diabetes on Liver Histology in Coastal Eastern India. Journal of Diabetes Mellitus, 2014;4:290-296.

7. Wai-Sun Wong $\vee$ et al. High prevalence of colorectal neoplasm in patients with non-alcoholic steatohepatitis. Gut doi:10.1136/gut.2011.237974

8. Hwang ST, Cho YK, Park JH, et al. Relationship of non-alcoholic fatty liver disease to colorectal adenomatous polyps. J Gastroenterol Hepatol. 2010;25:562-567.

9. Braun S, Bitton-Worms K, LeRoith D. The Link between the Metabolic Syndrome and Cancer. Int J Biol Sci. 2011;7:1003-1015.

10. Said O.Muhidin, Ahmed A.Magan, Khalid A. Osman, Shareef Syed, and Mohamed H. Ahmed. The Relationship between Nonalcoholic Fatty Liver Disease and Colorectal Cancer: The Future Challenges and Outcomes of theMetabolic Syndrome. J Obes. 2012:637538.

11. Trevisan M, Liu J, Muti P, Misciagna G, Menotti A, Fucci F. On behalf of the Risk Factors and Life Expectancy Research Group. Markers of insulin resistance and colorectal cancer mortality. Cancer Epidemiol Biomarkers Prev. 2001;10:937-941.

12. Charatcharoenwitthaya $P$, Lindor $K D$. Role of radiologic modalities in the management of non-alcoholic steatohepatitis. Clin. Liver Dis. 2007;11:37-54. 